\title{
A sala de aula invertida na percepção de estudantes de uma universidade pública brasileira
}

\author{
Marcelo Valério*, Joschua Rezende da Silva**, Gabrielly Giovana Pereira Senes ${ }^{\star * *}$, \\ William Junior do Nascimento****
}

\section{Resumo}

Resultados de pesquisas e de relatos de experiência transformaram a sala de aula invertida em tendência no ensino superior. Algumas críticas recentes, contudo, têm arrefecido o entusiasmo, reivindicando evidências mais amplas e contextualizadas. Este artigo buscou identificar e analisar a percepção de estudantes que vivenciaram a sala de aula invertida em uma universidade pública brasileira. Um questionário foi desenvolvido e aplicado aos alunos matriculados em sete disciplinas das áreas de ciência e tecnologia, em duas licenciaturas e três engenharias. As 122 respostas foram tratadas por estatística descritiva e analisadas comparativamente. Embora haja uma tendência de acolhimento, interesse e percepção positiva sobre a sala de aula invertida, os resultados não são homogêneos e se constatou um preocupante contingente de estudantes resistentes.

Palavras-chave: Metodologias ativas. Ensino superior. Ensino centrado no estudante.

* Doutor em Educação para a Ciência e a Matemática pela Universidade Estadual de Maringá. Docente da Universidade Federal do Paraná, atuando no Programa de Pós-Graduação em Educação em Ciências e em Matemática e nas Licenciaturas do campus avançado Jandaia do Sul. Brasil. E-mail: marcelovalerio@ufpr.br

* Licenciado em Computação pela Universidade Federal do Paraná. Brasil. E-mail: rezendejoschua@gmail.com

*** Licencianda em Ciências Exatas - Matemática pela Universidade Federal do Paraná. Brasil. E-mail: gabriellysenes@gmail.com

*t*** Doutor em Física pela Universidade Federal de São Carlos. Docente do Programa de Pós-Graduação em Ensino (Mestrado Profissional) da Universidade Estadual do Norte do Paraná. Docente da Universidade Federal do Paraná - campus avançado Jandaia do Sul. Brasil. E-mail: williamjn@ufpr.br

Recebido em: 21/03/2020 - Aceito em: 03/09/2020

https://doi.org/ 10.5335/rbecm.v4i1.10740

http://creativecommons.org/licenses/by-nc-nd/4.0 


\section{Introdução}

Sala de aula invertida é uma abordagem didática em ascensão no ensino superior, sobretudo na última década e nas áreas de ciência e tecnologia, que sugere o enfrentamento à tradição expositiva com propostas de ensino centradas nos estudantes (JOHNSON et al., 2014). Na sala de aula invertida, o tempo fora de sala é dedicado ao estudo prévio dos conteúdos a partir de materiais selecionados e/ou preparados pelos professores, quase sempre com a mediação de tecnologias digitais de informação e comunicação; enquanto o tempo em sala se destina basicamente a atividades de aplicação do conhecimento por meio das chamadas metodologias ativas (BERGMANN; SAMS, 2012; VALENTE, 2014; VALÉRIO; MOREIRA, 2018). Conforme explicam e defendem Hamdan et al. (2013, p.4), na sala de aula invertida:

[...] algumas lições são disponibilizadas fora do espaço de aprendizagem em grupo, usando vídeos ou outros tipos de materiais. O tempo de aula, então, fica disponível para os alunos se envolverem em atividades hands-on, colaborar com os seus pares, e avaliar seu progresso; para os professores, o tempo permite fornecer assistência individualizada, orientação e inspiração (HAMDAN et al., 2013, p.4).

As premissas pedagógicas costumeiramente associadas à sala de aula invertida apontam para o questionamento das pedagogias tradicionais que, por sua vez, legitimam e estimulam posturas passiva dos estudantes e o ensino transmissivo. Mas o referencial das chamadas metodologias ativas é amplo e difuso. Aparentemente, emprestam elementos da filosofia pragmatista de John Dewey (do movimento escolanovista) e das implicações educacionais da psicologia cognitivista para constituir proposições de filiação construtivista. No plano teórico, defendem um ensino centrado no aluno, com valorização pedagógica do erro, consideração dos saberes prévios dos estudantes, foco no diálogo, na ação coletiva e na mediação pedagógica do professor. Na prática, materializam-se e difundem-se em métodos como a instrução por pares (peer-instruction ou think-pair-share), aprendizagem baseada em problemas (problem based learning), estudos de casos (case-based-learning), grupos de trabalho colaborativo, aprendizagem por investigação, entre outros (MICHAEL, 2006; BERBEL, 2011; MAYER, 2012; ROCHA; LEMOS, 2014). Revisões da literatura, como as feitas Michael (2006), Hamdan et al. (2013) e Freeman et al. (2014), reúnem trabalhos evidenciando que tais abordagens incrementam o envolvimento, o pensamento crítico e o desempenho dos estudantes em diferentes níveis de ensino e contextos. 
Assim, a sala de aula invertida se apresenta como um arranjo didático que altera os tempos e relações pedagógicas, valendo-se dessas metodologias principalmente no encontro presencial. Mas, suas origens e motivações são diversas e imprecisas. A literatura indica que ela esteve inicialmente associada a preocupações como as diferenças nos ritmos de aprendizagem dos alunos e com os chamados "estilos de aprendizagem". Nesse ponto, o trabalho de Lage et al. (2000) seria o pioneiro, ao relatar uma experiência na universidade de Ohio, Estados Unidos, em uma disciplina de Microeconomia, em 1996. Também naquele país, mas agora na educação básica, outra experiência é frequentemente apontada como desbravadora: dois professores de Química, preocupados com alunos ausentes ou afastados das atividades presenciais, começaram a gravar e disponibilizar na web vídeos de suas aulas, em 2007. Em livro publicado em 2012 eles relatam como esses vídeos transformaram suas aulas em ambientes de discussão aberta e aprendizagem ativa, guiadas pelo estudo prévio de seus alunos (BERGMANN; SAMS, 2012). O interesse pela sala de aula invertida explodiu, no entanto, quando em 2009 um professor Física da universidade de Harvard publicou um artigo na revista Science sugerindo o fim da aula expositiva. O professor Eric Mazur relatou de maneira breve e eloquente que ao invés de ensinar falando, agora ensinava questionando! Segundo ele, seus alunos deveriam apenas "ler o material antes de vir para a aula, de modo que o tempo em sala fosse dedicado às discussões, interações entre pares, e ao tempo para assimilar e pensar" (MAZUR, 2009, p.51).

Tal modelo tem sido amplamente difundido a partir de resultados obtidos em pesquisas em universidades estadunidenses, as quais dão conta de incremento nos índices de participação, engajamento, motivação, frequência, desempenho e domínio do conteúdo pelos estudantes. Além disso, há também ponderações teóricas sobre o desenvolvimento de habilidades profissionais, pensamento crítico, bem como as possibilidades de customização do ensino aos ritmos de aprendizagem e do valor da aprendizagem colaborativa que se desenvolve entre os estudantes (BERRETT, 2012; ARONSON; ARFSTROM, 2013; HAMDAN et al., 2013).

A repercussão da sala de aula invertida em países como o Brasil, onde já há um consórcio ${ }^{1}$ de instituições promovendo e implementando o modelo, merece, então, atenção pedagógica e esforços de pesquisa acadêmica. Trabalhos como de Francisco e Oliveira (2016) ou Caron-Cambraia e Pagani-Benvenutti (2018) exemplificam sua já propagação, inclusive, nos cursos de formação de professores. Mas singularida- 
des do fenômeno educativo em cada contexto sugerem o risco de vieses culturais, reivindicando que a adoção da sala de aula invertida em universidades brasileiras não se dê somente a partir de evidências colhidas em outros cenários, mas que ela própria seja também palco das investigações. A tradição de pesquisa sobre o tema demonstra que ainda há poucos estudos nacionais cotejando os resultados com a literatura internacional, os quais poderiam começar a constituir evidência orientadora da prática pedagógica para os docentes brasileiros e formuladores de políticas pedagógicas institucionais (LEITE, 2006; BIZOLATTI; COELHO-NETO, 2018; VALÉRIO; BELETI-JUNIOR, 2019).

Assim justificado, e preocupado também com algumas críticas que começam a surgir sobre os desafios do autodidatismo ou sobre o possível caráter antidemocrático do modelo (VALÉRIO; MOREIRA, 2018), este artigo tem por objetivo identificar e analisar as percepções de universitários brasileiros que vivenciaram disciplinas de graduação onde a sala de aula invertida foi adotada como abordagem privilegiada

\section{Os achados da literatura sobre a sala de aula invertida}

O maior volume de pesquisas empíricas e relatos e experiência sobre a sala de aula invertida emanam das áreas de ciência e tecnologia, onde a proposta tem sido usada para questionar valor e a força da tradição pedagógica de aulas expositivas (GIANNAKOS; KROGSTIE; CHRISOCHOIDES, 2014; ZAINUDDIN; HALILI, 2016). Além disso, o impacto econômico e social dessas áreas também contribui para que haja pressão política e pedagógica em torná-las mais atraentes e mais capazes de gerar sucesso acadêmico entre os estudantes (BRADFORD et al., 2015; WALDROP, 2015).

Revisões de literatura, que reúnem e analisam os achados desses trabalhos, sustentam haver prevalência de resultados positivos (BISHOP; VERLEGER, 2013; ABEYSEKERA; DAWSON, 2014; GIANNAKOS; KROGSTIE; CHRISOCHOIDES, 2014; HUBER; O'FLAHERTY; PHILLIPS, 2015; WERNER, 2016). As experiências e investigações sobre a sala de aula invertida registram haver interações mais frequentes e producentes entre professores e alunos, e entre os próprios alunos (WIEMAN, 2011; BERGMANN; SAMS, 2012; BROWN, 2012; DESLAURIERS; SCHELEW; DRISCOLL, 2012; STRAYER, 2012; GALINDO; QUINTANA, 2016; OFUGI, 2016); maior oportunidade de trabalho individualizado, adequado ao ritmo 
dos estudantes (LAGE; PLATT; TREGLIA, 2000; BROWN, 2012; DRISCOLL, 2012; JOHNSON, 2013; OGDEN, 2015); incremento em habilidades comunicativas, de pensamento crítico e resolução de problemas (GOMEZ, 2016; TURAN; GOKTAS, 2016; TAZIJAN et al., 2017); e melhora significativa no clima de aprendizagem, desempenho e frequência dos discentes (MAZUR, 2009; PAPADOPOULOS; ROMAN, 2010; DESLAURIERS; SCHELEW; WIEMAN, 2011; MARLOWE, 2012; TREVELIN; PEREIRA; OLIVEIRA NETO, 2013; BUSATO et al., 2016; NOURI, 2016; SENGEL, 2016; KIM; KIM, 2017; OLAKANMI, 2017).

A repercussão midiática da sala de aula invertida se explica pela profusão desses resultados promissores, claro, mas sua adoção e implementação nos diferentes contextos não prescinde de parcimônia e crítica. Dentro da própria literatura acadêmica e tradição de pesquisa sobre o tema, já começam a surgir questionamentos: para Jensen, Kummer e Godoy (2015) e DeLozier e Rhodes (2016), os benefícios apontados não decorrem necessariamente da "inversão" e do estudo prévio, mas principalmente das características de aprendizagem ativa do encontro presencial; para Sletten (2017), não há correlação entre a preferência por uma aprendizagem ativa em sala e o comprometimento com os estudos fora dela; para DeLozier e Rhodes (2016) e Menella (2016), não se deve assumir a percepção dos estudantes como métrica adequada e direta para retratar a aprendizagem. Ou seja, permanece questionável qual a capacidade de autogerência do aprendizado pelos estudantes fora de sala de aula (SLETTEN, 2017); ou, para que conteúdos, disciplinas e perfis discentes o modelo se faz adequado (STRAYER, 2012); e mesmo, se não seria a sala de aula invertida um esforço para condensar ou resumir um currículo inchado e ineficaz (BERRETT, 2012; BOGOST, 2013). Estas e outras advertências sobre a sala de aula invertida estão detalhadas na compilação de críticas feita por Valério e Moreira (2018).

Mesmo assim, a sala de aula invertida está na pauta do dia, sobretudo no ensino superior. Em um tempo em que as tecnologias de informação e comunicação contribuem para tornar obsoletas as aulas expositivas tradicionais, elas também se apresentam como potenciais ferramentas para hibridizar ensino presencial e a distância (VALENTE, 2014). A viabilização de práticas de ensino como estudos de caso ou simulações (role playing games), de relações colaborativas de ensino entre os estudantes, de avaliações que revelam mais rápida e claramente as dificuldades dos alunos, permitindo intervenções adequadas, são certamente atraentes. Por 
outro lado, a adoção açodada e instrumental da sala de aula invertida pode ser um caminho arriscado para professores, estudantes e instituições. Aumentar o volume e a frequência do estudo, externo aos ambientes de ensino, também pode ser ineficaz e antidemocrático. É por isso que investigações sobre o potencial e o valor da adoção dessas estratégias precisam ser estimuladas, considerando também que sua compreensão dar-se-á necessariamente à luz de cada contexto de ensino e ambiente de aprendizagem que a sala de aula invertida estimular ou promover.

No ensino superior brasileiro, os esforços de pesquisa empírica e os relatos de experiência sobre a sala de aula invertida recém começaram a repercutir. Empreender uma análise quantitativa sobre a percepção dos estudantes, tradição em outros países, permite estabelecer paralelos entre os achados da literatura internacional e o contexto nacional, constituindo evidências que podem contribuir para orientar o trabalho de professores e instituições de ensino superior brasileiras.

\section{O cenário de pesquisa e os encaminhamentos metodológicos}

Durante o primeiro semestre letivo do ano de 2017, cinco docentes aplicaram metodologias ativas, estruturadas no modelo sala de aula invertida, em sete disciplinas do núcleo comum de cinco cursos de graduação, conforme a Tabela 1 . Especificamente, em Geometria Analítica, Microbiologia Geral e Arquitetura de Computadores, aproximadamente um terço das aulas foram consideradas como invertidas, definindo uma implantação parcial. Nas outras disciplinas, mais de dois terços dos encontros do semestre ocorreram dentro da proposta (considerada, portanto, uma implantação total). 
Tabela 1: Distribuição e periodização das 7 disciplinas, em 8 edições, em 5 cursos de graduação da instituição.

\begin{tabular}{|c|c|c|}
\hline Disciplinas & Periodização & Cursos \\
\hline Geometria Analítica & $1^{\circ}$ & \multirow[t]{3}{*}{ Licenciatura em Ciências Exatas (LCE) } \\
\hline Física I & $1^{\circ}$ & \\
\hline Química III & $3^{\circ}$ & \\
\hline Física II & $3^{\circ}$ & Engenharia de Produção (EPR) \\
\hline Física II & $3^{\circ}$ & Engenharia Agrícola (EAG) \\
\hline Microbiologia Geral & $5^{\circ}$ & \multirow[t]{2}{*}{ Engenharia de Alimentos (EAL) } \\
\hline Química Orgânica & $5^{\circ}$ & \\
\hline $\begin{array}{l}\text { Arquitetura de } \\
\text { Computadores }\end{array}$ & $3^{\circ}$ & Licenciatura em Computação (LCO) \\
\hline
\end{tabular}

Fonte: Elaborado pelos autores.

Um mesmo professor foi responsável pelas três Físicas, outro pelas duas Químicas, enquanto as demais disciplinas tiveram, cada qual, um professor específico. Todos os docentes relatam ter orientado o estudo prévio dos conteúdos pelos estudantes utilizando, majoritariamente, videoaulas disponíveis na rede web (selecionadas em plataformas como a Khan Academy) e notas de aula próprias enviadas via e-mail ou aplicativos de mensagens. Em sala, desenvolviam a resolução guiada de exercícios ou a resolução de problemas em grupos, recorrendo a estratégias interativas e colaborativas como a peer-instruction ou think-pair-share (LYMAN, 1987; MAZUR, 1997). Em uma aproximação ao que Novak et al. (1999) descreve como just-in-time teaching (JiTT), faziam pequenas explanações teóricas pontuais enquanto acompanhavam as atividades dos estudantes, adicionando questões previamente elaboradas e repercutindo as dificuldades que identificavam.

Todos também fizeram breves revisões de parte dos conteúdos estudados ao início das aulas, atendendo a pedidos dos próprios estudantes. Além disso, dois professores (o que ministrou as disciplinas de Física I e II, e o que ministrou Geometria Analítica) utilizaram ainda recursos e dispositivos de tecnologia digital para recolher as respostas dos estudantes a testes feitos em casa, previamente, ou para analisar as respostas em tempo real, em sala (formulários online e/ou sistemas de resposta a audiência (clickers) - experiência relatada com maiores detalhes em Valério et al. (2019). 
Enquanto as disciplinas eram ministradas, os pesquisadores dedicaram esforços no acompanhamento e no estudo da literatura sobre a sala de aula invertida no Ensino Superior, destacando pesquisas empíricas onde a percepção dos estudantes também fosse objeto de estudo. Desses estudos derivaram cinco pautas de interesse: (1) Acolhidas ou resistências à proposta didática da sala de aula invertida; (2) Percepção sobre as relações pedagógicas de interação professor-alunos e alunos-alunos; (3) Padrões de motivação e o engajamento em disciplinas que adotam o modelo; (4) Valorização ou enfrentamento do momento de estudo prévio; (5) Avaliação da experiência com a sala de aula invertida.

Mas, embora estes elementos fossem recorrentes, eles se apresentavam de modo particular ou disperso nos estudos, não tendo sido localizado nenhum instrumento de coleta de dados que os contemplasse em conjunto. Assim, mobilizados pelo desafio do viés cultural (pesquisas estrangeiras) e pela inadequação, incipiência ou carência dos instrumentos metodológicos na produção nacional, decidiu-se pelo desenvolvimento de um questionário próprio. Foram consideradas, para tanto, as peculiaridades do contexto em análise, as possibilidades de operacionalização da pesquisa e as orientações metodológicas de Vieira (2009) e Moreira (2011).

Nesta perspectiva, buscando um instrumento capaz de revelar as percepções, o engajamento e o interesse dos estudantes em questão, fora desenvolvido um questionário (anexo I) com dezessete (17) itens (perguntas), sendo dois itens de identificação (informação sobre a disciplina cursada e se correspondia ao primeiro contato com essa proposta, respectivamente), um de avaliação direta da disciplina que cursou (em uma escala de zero a dez) e doze questões fechadas, estruturadas mediante asserção de valor em uma escala Likert de sete pontos. Outras duas questões eram abertas e buscaram complementar os dados quantitativos, solicitando uma declaração formal da percepção de vantagens e desvantagens pelos estudantes - mas são alvo de uma análise particular, de abordagem qualitativa.

A validação da estrutura e coerência dos itens foi feita em colaboração de um docente da área de Estatística. Para as questões fechadas, a confiabilidade desse questionário foi analisada com a medida de correlação alfa de Cronbach (FREITAS; RODRIGUES, 2005; VIEIRA, 2009), resultando em um índice de 0,93. Além disso, uma análise semântica, evitando ambiguidades, má compreensão ou formulações inapropriadas foi feita pelos próprios professores (ministrantes das disciplinas), a convite da equipe de pesquisa (validade de face). 
Quando do encerramento do semestre letivo os estudantes foram convidados a responder ao questionário elaborado. Para participação na pesquisa, foram selecionados todos os estudantes que concluíram as referidas disciplinas, com aproveitamento acadêmico ou não (aprovados ou reprovados). Estudantes reprovados por frequência ou desistentes não foram considerados. O número de matrículas nas sete disciplinas (oito turmas) e, consequentemente, o universo amostral foi de duzentos e sete estudantes (207). Destes, trinta e quatro (34) estavam matriculados em duas disciplinas, e sete (7) em três disciplinas, tornando a população amostrada menor: cento e sessenta e seis $(\mathrm{N}=166)$ estudantes. Após busca direta e eletrônica (e-mail), definiu-se uma amostra efetiva de cento e vinte e duas $(n=122)$ (total de respostas obtidas aos questionários). No entanto, como alguns estudantes responderam mais de uma vez o questionário (apresentaram respostas para duas ou três disciplinas em que estavam matriculados), deve-se considerar 87 respondentes (unidades amostrais). Assim, a representatividade da amostra ficou em 52\%, considerando o número de estudantes ( 87 respondentes em 166 pessoas). Todos os respondentes consentiram de modo autônomo e esclarecido com sua participação, tendo seu anonimato garantido conforme consignado em documento específico. Na Tabela 2 constam as disciplinas em análise, bem como a distribuição de seus estudantes na amostra.

Tabela 2: Distribuição e representatividade dos estudantes participantes da pesquisa, por disciplina.

\begin{tabular}{|l|l|l|}
\hline Disciplina & $\begin{array}{l}\text { No de respostas amostradas } \\
\text { (\% do total de alunos aptos } \\
\text { a responderem o } \\
\text { questionário na disciplina) }\end{array}$ & $\begin{array}{l}\text { Percentual da } \\
\text { amostra total }\end{array}$ \\
\hline Arquitetura de Computadores & $7(47 \%)$ & $5,74 \%$ \\
\hline Física I & $17(59 \%)$ & $13,93 \%$ \\
\hline Física II (EPR) & $34(57 \%)$ & $27,87 \%$ \\
\hline Física II (EAG) & $11(36 \%)$ & $9,02 \%$ \\
\hline Geometria Analítica & $21(66 \%)$ & $17,21 \%$ \\
\hline Microbiologia Geral & $10(65 \%)$ & $8,20 \%$ \\
\hline Química Orgânica & $12(87 \%)$ & $9,83 \%$ \\
\hline Química III & $10(77 \%)$ & $8,20 \%$ \\
\hline TOTAL DE QUESTIONÁRIOS & $\mathbf{1 2 2}$ & $\mathbf{1 0 0} \%$ \\
\hline
\end{tabular}

Fonte: Elaborado pelos autores. 
A disciplina com maior representação na amostra é Física II, do curso de Engenharia de Produção, totalizando 27,87\% das respostas; e a menor, Arquitetura de Computadores, que representou $5,74 \%$ da amostra. As taxas de respostas dentro das próprias disciplinas foram quase sempre superiores a $50 \%$ dos estudantes concluintes - as exceções foram a Física II, onde a porcentagem de alunos respondendo a pesquisa foi de $36 \%$ e Arquitetura de Computadores, com $47 \%$.

As doze questões fechadas foram alvo de uma análise quantitativa, valendo-se de estatística descritiva, destacando os resultados de média (na escala Likert de sete pontos, com valores entre 1 e 7), de desvio padrão e dos percentis extremos (soma das opções "1 mais 2", e "6 mais 7"), em cada uma das questões. Os resultados foram descritos e sua interpretação focalizou a busca por padrões ou tendências, considerando as asserções de valor dentro da escala utilizada; e a partir de contrastes entre as disciplinas. As respostas acumuladas nos percentis extremos, para efeito da discussão, foram assumidas como asserções negativas ou positivas. Os resultados foram ajustados às cinco grandes pautas teóricas já citadas, com vistas a uma descrição e discussão mais sintética e coesa.

\section{Resultados e discussões}

De modo geral, os participantes dessa pesquisa exibem tendência de aceitação, interesse e percepção positiva sobre a sala de aula invertida, ainda que $72 \%$ das respostas tenham informado estarem vivenciando pela primeira vez tal proposta didática. Na escala de asserção de valor, aproximadamente $38 \%$ dos estudantes reconhecem haver aspectos positivos decorrentes da estratégia (assinalam 6 ou 7); enquanto próximo de $15 \%$ das respostas se encaminharam para uma percepção negativa da abordagem (assinalam 2 ou 1).

Considerando as doze questões fechadas, nas quais foram utilizadas a escala Likert, a média geral assinalada foi de 4,74 (para um mínimo de 1 máximo de 7). A Tabela 3 apresenta os resultados, individualizados por questão (Q3, Q4, ..., Q13 e Q17), considerando a média, o desvio padrão e o agrupamento dos percentis dos extremos da escala. 
Tabela 3: Estatística descritiva dos resultados das doze questões fechadas do questionário

\begin{tabular}{|l|l|l|l|l|}
\cline { 2 - 5 } \multicolumn{1}{c|}{} & Média & Desvio padrão & Agrupamento 1+2 & Agrupamento 6+7 \\
\hline Q3 & 4,9 & 1,29 & $7,30 \%$ & $40,10 \%$ \\
\hline Q4 & 4,4 & 1,69 & $21,30 \%$ & $31,10 \%$ \\
\hline Q5 & 4,3 & 1,71 & $22,90 \%$ & $31,10 \%$ \\
\hline Q6 & 4,8 & 1,71 & $18,80 \%$ & $40,10 \%$ \\
\hline Q7 & 5 & 1,24 & $6,50 \%$ & $31,10 \%$ \\
\hline Q8 & 4,6 & 1,73 & $13,90 \%$ & $39,30 \%$ \\
\hline Q9 & 4,7 & 1,55 & $10,60 \%$ & $34,40 \%$ \\
\hline Q10 & 4,5 & 1,76 & $26,20 \%$ & $31,90 \%$ \\
\hline Q11 & 5,04 & 1,52 & $8,10 \%$ & $45,90 \%$ \\
\hline Q12 & 4,8 & 1,79 & $13,90 \%$ & 43,40 \\
\hline Q13 & 5,3 & 1,65 & $10,60 \%$ & $56,50 \%$ \\
\hline Q17 & 4,5 & 1,77 & $18,80 \%$ & $36,80 \%$ \\
\hline
\end{tabular}

Fonte: Elaborado pelos autores.

Tendo em vista a heterogeneidade do número amostral apresentado anteriormente na Tabela 2, bem como a premissa teórica de que o ensino é uma atividade singular e contingenciada, para cada uma das questões, ainda, os resultados foram segmentados por disciplinas e contrastados. Os principais achados estão descritos abaixo, organizados e ajustados em cinco grandes temas:

\section{Interesse e percepção da sala de aula invertida:}

Os estudantes avaliaram positivamente a proposta conceitual da sala de aula invertida, baseada no estudo prévio, seguido pelo trabalho ativo em sala. Somente 7\% do total de estudantes se manifestou resistente à ideia, assinalando as opções 1 e 2 da escala de 7 pontos. Esses resultados estão em consonância com o que foi percebido na maior parte dos estudos sobre o tema - de acordo com importante revisão de literatura feita por Giannakos, Krogstie e Chrisochoides (2014).

Tal percepção, no entanto, não foi homogênea quando as disciplinas foram comparadas. Disciplinas mais avançadas, como Microbiologia Geral, em Engenharia de Alimentos, e Química III, na Licenciatura em Ciências Exatas, tiveram índices mais altos de acolhimento (80\% e 75\%, respectivamente). Química Orgânica, também avançada, no entanto, teve um padrão de acolhimento semelhante ao de Geometria Analítica, vivenciada por alunos iniciantes. E, sugerindo não haver mesmo consen- 
so, nas disciplinas de Física foi significativo o registro de um grupo de estudantes resistentes às proposições da sala de aula invertida, enquanto em Arquitetura de Computadores mais de $85 \%$ dos estudantes ocupou o centro da escala (assinalando entre 3 e 5), demonstrando incerteza quanto à proposta.

É possível e provável que variações na qualidade das relações pedagógicas contribuam com esses resultados pouco homogêneos (LEITE, 2006; STRAYER, 2012).

\section{Motivação e engajamento}

O impacto da sala de aula invertida na motivação para frequentar e participar das aulas não foi comunal: se pouco mais de $30 \%$ dos estudantes reconhecem maior motivação, aproximadamente $23 \%$ declaram não ter se sentido mais motivados. Quando as disciplinas foram comparadas, os resultados se mostraram bastantes desiguais. Em Microbiologia, parcela expressiva dos estudantes confirmam maior motivação (80\%), não havendo negativas a respeito. Por outro lado, em Arquitetura de Computadores, nenhum estudante assinalou haver mais ou maior motivação para com as aulas. As piores percepções apareceram entre os estudantes de Física I e II, onde quase um terço das classes declarou não ter sua motivação influenciada pela sala de aula invertida.

Torna-se necessário estudar caso-a-caso o fenômeno da motivação dos estudantes, ao passo que se reproduzem aqui uma das conclusões da revisão feita por Karabulut-Ilgu, Cherrez e Jahren (2017), que sinaliza que o fenômeno de aumento da frequência não pode ser assumido como consenso quando se fala em adoção da sala de aula invertida.

Em relação ao tempo de estudo, considerando toda a amostra de estudantes, $40 \%$ relatam ter estudado mais por ocasião da sala de aula invertida, enquanto $19 \%$ sugerem não ter aumentado seu tempo dedicado aos estudos. Nas duas Químicas e em Microbiologia, mais da metade dos estudantes respondentes assumem ter estudado mais em função da experiência. Mas, enquanto os estudantes de Química III sinalizam aumento significativo de seu tempo de estudo (83\%), em Arquitetura de Computadores se confirma o maior percentual de estudantes declarando não reconhecer tal impacto em seu comportamento (43\%).

Não parece possível estabelecer justificativa direta para o engajamento da maior parte dos estudantes, mas como relata Sengel (2016), a maior parte dos alunos pare- 
cem perceber que precisam passar mais tempo estudando, caso contrário, encontram ainda mais dificuldades para acompanhar os tópicos trabalhados em sala de aula.

\section{Estudo prévio: compromisso e auto-regulação}

Poucos estudantes da amostra assumem não ter se dedicado ao estudo prévio conforme orientado $(6,5 \%)$, havendo $31 \%$ declarando que sempre ou quase sempre o fizeram. Em todas as disciplinas o contingente de estudantes que dizem ter seguido as orientações e estudado previamente é significativo (médias acima de 5,2). Em Física II (ambos os cursos) e em Arquitetura de Computadores estiveram os estudantes que se declararam mais reticentes ao estudo prévio conforme o professor sugeriu. Peculiar, ainda, foi o resultado desta última: a disciplina contou com o maior percentual de alunos resistentes ao estudo prévio (14\%) e o segundo maior percentual de estudantes comprometidos com essa atividade (43\%). Esses resultados se assemelham aos de outros estudos sobre a percepção de estudantes, no Brasil (GOMES et al., 2010) e no exterior (MCNALLY et al., 2017), que também identificaram opiniões bastante discrepantes.

Nos trabalhos analisados por Giannakos, Krogstie e Chrisochoides (2014) e por Huber e Werner (2016), a maior parte dos alunos recebem bem a ideia de terem acesso prévio aos materiais, estabelecer ritmos próprios de estudo e aprender de modo mais independente. Musallam (2010) sustenta que o estudo prévio potencializa a aprendizagem presencial posterior. Os alunos de Álgebra estudados por Ogden (2015) sugerem que o arranjo didático com os vídeos antecipados os concedeu a oportunidade de se auto-instruir e promoveram o aumento de sua aprendizagem. Nesse sentido, os estudantes aqui analisados corroboram a literatura. Somente $14 \%$ sugerem que antecipar o estudo não potencializou sua compreensão e domínio do conteúdo, enquanto $39 \%$ apontaram haver impacto do estudo prévio nesse processo. Destacadamente, em Microbiologia Geral, 90\% dos estudantes dizem ter dominado e compreendido melhor o conteúdo por conta da experiência.

Importa, entretanto, que fenômeno inverso ocorreu com 57\% dos alunos da Arquitetura de Computadores, para os quais a relação estudo prévio e domínio/ compreensão de conteúdos não se revelou. Nas disciplinas de Física II, por sua vez, a relação foi incerta, havendo estudantes para os quais o estudo prévio foi positivo, e, outros, para os quais a antecipação dos estudos não surtiu o mesmo efeito. 
Já a característica dos materiais enviados previamente pelos professores não foi assumida como um empecilho ou obstáculo para os estudantes analisados: a maior parte deles (46\%) reconheceu que os materiais estavam adequados às suas capacidades de aprendizagem, e somente $8 \%$ discordou. Essa tendência se evidenciou em todas as disciplinas, inclusive em Arquitetura de Computadores - que apresenta os dados menos alvissareiros. Particularmente, os estudantes de Física II, da Engenharia de Produção, foram os que assumiram maiores dificuldades para lidar com o material de estudo prévio.

\section{Encontro presencial: interações pedagógicas e ritmo de aprendizagem}

No que tange às metodologias utilizadas em sala de aula, aproximadamente um terço dos estudantes se manifestaram receptivos, enquanto $11 \%$ não perceberam nelas potencial para incrementar o domínio e compreensão dos conteúdos. A Microbiologia, novamente, foi a disciplina onde houve maior acolhimento das propostas desenvolvidas pelo professor (80\%), seguida por Geometria Analítica (62\%). Tais resultados corroboram a indicação de que os estudantes percebem estar aprendendo mais rápido e melhor na sala de aula invertida (OGDEN, 2015; BUSATO et al., 2016) e, portanto, valorizam as estratégias ativas (SLETTEN, 2017). Apenas em Física II, da Engenharia Agrícola, houve menos estudantes referendando as propostas que criticando, mas, de modo interessante, a mesma disciplina no curso de Engenharia de Produção teve um índice de acolhimento às propostas relativamente alto (45,5\%), bastante parecido com Química III $(41,6 \%)$.

Revisões de literatura também dão conta de que a sala de aula invertida exige que os estudantes trabalhem de modo colaborativo, aumentando níveis de envolvimento por meio de debates e discussões (GIANNAKOS; KROGSTIE; CHRISOCHOIDES, 2017; KARABULUT-ILGU; CHERREZ; JAHREN, 2017). Sobre esse tema, aproximadamente um terço dos estudantes declarou ter havido mais e melhores interações entre os estudantes, e, desses, com o professor. Não perceberam igualmente tais benefícios $21 \%$. Novamente, Microbiologia aparece com o principal resultado mais positivo, enquanto a Arquitetura de Computadores como o principal resultado negativo. Merece atenção, ainda, que a disciplina de Física II, lecionada pelo mesmo docente e com a mesma periodização, quando ministradas para turmas de cursos diferentes, definiu uma percepção também distinta sobre as interações: enquanto os estudantes da Engenharia Agrícola reclamaram, os da Engenharia 
de Produção reconheceram ter havido mais e melhores interações alunos-alunos e alunos-professor.

Quando questionados sobre se os professores estiveram mais atentos aos seus ritmos e dificuldades, os estudantes não estabeleceram uma tendência. A média geral foi de 4,5, mas o contingente de estudantes que registrou posição em um ou outro extremo da escala foi similar. A análise dos resultados por disciplina, contudo, permite estabelecer um rol onde os estudantes não reconheceram tal comportamento em seus professores, composto pela Arquitetura de Computadores e as três Físicas, e outro, no qual os estudantes reconheceram com mais clareza perceber uma postura mais atenta do professor, definido pelas Químicas, Microbiologia e Geometria Analítica.

Trata-se, este, de um resultado destacado da pesquisa, que permite inferir que a percepção da qualidade nas relações professor-aluno reflete diretamente na percepção de outros elementos da experiência didática dos estudantes. Autores como Jensen, Kummer e Godoy (2015) e DeLozier e Rhodes (2016) vêm questionando o poder transformador da sala de aula invertida, sugerindo que os benefícios encontrados derivam não do estudo prévio, mas, e sobretudo, das características de aprendizagem ativa do encontro presencial.

Importa, neste ponto, salientar que não apenas os estudantes, mas também os professores das disciplinas em análise, eram, em sua maioria, inexperientes em relação à sala de aula invertida. Trabalhos recentes, como o de Suhr (2016), Pavanelo e Lima (2017), Serqueira (2017) e Valério (2018) enfatizam justamente o desafio técnico e epistemológico enfrentado pelos professores ao alterar suas práticas e posturas pedagógicas, devendo-se ter em conta também essa contingência em estudos sobre a percepção dos estudantes.

\section{Avaliação da experiência com a sala de aula invertida nas disciplinas}

A maior parte dos estudantes avalia que seu desempenho foi compatível com sua dedicação, capacidade e compromisso durante o semestre (43\% assinalaram o alto da escala). Com exceção à Arquitetura de Computadores e à Física I, em todas as outras disciplinas a porcentagem de estudantes que reconhecem como coerente a relação entre seu desempenho e as variáveis sinalizadas é maior. Considerando apenas as Químicas, a Microbiologia Geral e a Geometria Analítica, a percepção dos estudantes é bastante positiva. Nas disciplinas de Física II, contudo, chama a 
atenção as altas taxas de discordância em relação ao tema, ou seja, parcela significativa dos estudantes entende que sua capacidade e dedicação durante o semestre não foi condizente com seu desempenho.

A maior parte dos estudantes (57\%) também reconheceu como justos e adequados os critérios e as formas de avaliação adotadas em todas as disciplinas. Os resultados mais baixos foram registrados nas Físicas e Arquitetura de Computadores, mas mesmo nessas disciplinas a percepção de justiça e adequação da avaliação esteve acima dos $40 \%$ dos estudantes.

Quando avaliadas, todas as disciplinas receberam notas superiores a 5, em uma escala de 10 pontos. A disciplina melhor avaliada foi Microbiologia Geral, com média 9, seguida por Química III (média 8) e Geometria Analítica (média 7) - disciplinas de quinto, terceiro e primeiro período, respectivamente. Em Geometria Analítica, contudo, diferente das duas anteriores, o alto desvio padrão sugere haver alunos avaliando mal a disciplina. As médias mais baixas não estiveram muito distantes, ficando entre 5,45 (Física II, na Engenharia de Produção) e 6,54 (Arquitetura de Computadores). Nestas, também, o desvio revela a percepção pouco homogênea dos estudantes. Embora mais modestos, esse padrão de avaliação positiva das disciplinas sala de aula invertida reverberam os achados de outras experiências nacionais em áreas correlatas, como a de Pavanelo e Lima (2017), em Cálculo, em cursos de Engenharia do Instituto Tecnológico da Aeronáutica.

Por fim, questionados se estariam interessados e dispostos em repetir vossas experiências com a sala de aula invertida, pouco mais de um terço dos estudantes analisados manifestou-se positivamente. Índices de resistência maiores foram encontrados entre os alunos iniciantes da Licenciatura em Ciências Exatas, que cursaram Física I e Geometria Analítica. Seus colegas que estavam mais avançados no curso, na disciplina de Química III, consignaram bem menos resistência e muito maior intenção de repetir a experiência em outras disciplinas. Em Arquitetura de Computadores os estudantes se mostraram bastante divididos, e em Física II a percepção foi distinta quando se comparou as duas turmas das engenharias. Tal incerteza pode repercutir a proposição de Sletten (2017), que salienta não haver relação clara e direta entre a acolhida dos estudantes pela estratégia e seus comportamentos de auto-regulação da aprendizagem, ou seja, mesmo o estudante que acolha a sala de aula invertida pode se mostrar incapaz de dar conta dos desafios que ela propõe. 


\section{Conclusões e implicações de pesquisa}

Essas análises permitem inferir que a adoção e implementação da sala de aula invertida não demarca transformação pedagógica homogênea e democrática, necessariamente. Os resultados variam bastante quando as diferentes disciplinas são comparadas, sugerindo que outros aspectos - relacionados às relações pedagógicas professores-estudantes ou aos encaminhamentos metodológicos - podem e devem ser decisivos.

Em diálogo com a literatura, os achados dessa pesquisa aderem à preocupação de Strayer (2012), para quem, talvez, o modelo sala de aula invertida não seja o mais adequado para os cursos introdutórios - como é o caso da maior parte da amostra de estudantes. É possível, ainda, que a falta da cultura de comprometimento dos estudantes brasileiros constitua também um desafio para a sala de aula invertida, como propõe Suhr (2016), fazendo com que novos e aprofundados estudos precisem seguir sendo feitos, sobretudo com abordagens capazes de revelar mais claramente as peculiaridades de cada situação e contexto de ensino.

Nesse estudo, nem a periodização das disciplinas, nem o padrão de adoção (parcial ou total), nem o curso específico, nem um professor em particular pôde ser assumido como variável definidora de um padrão de resultados. Os estudantes parecem acolher as metodologias ativas associadas à sala de aula invertida e exibir uma percepção positiva desse modelo didático, mas a existência de um significativo contingente de estudantes resistentes ou relutantes, bem como os resultados modestos em algumas disciplinas, deve ser suficiente para conter possíveis euforias e orientar a necessidade de novas pesquisas.

O caráter contingencial, histórico e social, do ensino, não permite referendar a adoção de arranjos metodológicos como soluções transformadoras, por si mesmas. 


\section{flipped classroom approach in a brazilian public university}

\section{Abstract}

The flipped classroom approach has been described as a trend in higher education. Some recent criticisms, however, have attenuated enthusiasm, claiming for a broader and more contextualized evidence. This article aimed to identify and analyze the perception of students who experienced the flipped classroom in a Brazilian public university. A questionnaire was developed and applied to the students enrolled in seven science and technology disciplines, in two undergraduate licentiate and three engineering courses. Descriptive statistics was used to treat and analyze the 122 responses. Although there was a tendency of acceptance, interest and positive perception about the flipped classroom, the results were not homogeneous and showed a worrying contingent of resistant students.

Keywords: Active learning. Higher education. Student-centered learning.

\section{Nota}

1 Ver http://sthembrasil.com/ para conhecer sobre o consórcio entre Instituições de Ensino Superior brasileiras e a LASPAU, descrito como uma iniciativa para o desenvolvimento da inovação acadêmica por meio do "Programa Acadêmico e Profissional para as Américas", afiliado à Universidade de Harvard.

\section{Rerências}

ABEYSEKERA, Lakmal; DAWSON, Phillip. Motivation and cognitive load in the flipped classroom: definition, rationale and a call for research. Higher Education Research \& Development, 34(1). p. 1-14, 2014.

BERBEL, Neusa Aparecida Navas. As metodologias ativas e a promoção da autonomia de estudantes. Semina: Ciências Sociais e Humanas, Londrina, 32(1), p. 25-40, 2011.

BERGMANN, Jonathan; SAMS, Aaron. Flip Your Classroom: reach every student in every class every day. Eugene, Oregon: Iste, 2012.

BERRETT, Dan. How flipping the classroom can improve the traditional lecture. Chronicle of Higher Education, 19, 2012.

BISHOP, Jacob Lowell.; VERLEGER, Mathew A. The Flipped Classroom: A Survey of the Research. Proceedings of the Annual Conference of the American Society for Engineering Education, 6.219, 2013.

BIZOLATTI, Aline da Silva; COELHO-NETO, João. Sala de Aula Invertida: possíveis aproximações para o ensino da Matemática. Revista Thema, v. 15, n. 3, p. 848-859, 2018.

BOGOST, Ian. The Condensed Classroom: "Flipped” classrooms don't invert traditional learning so much as abstract it. The Atlantic, 2013. Disponível em: https://www.theatlantic.com/technology/ archive/2013/08/the-condensed-classroom/279013/. Acesso em: 01/03/2017. 
BRADFORTH, Stephen E.; MILLER, Emily R.; DICHTEL, William R.; LEIBOVICH, Adam K.; FEIG, Andrew L.; MARTIN, James D.; BJORKMAN, Karen S.; SCHULTZ, Zachary D.; SMITH, Tobin L. Improve undergraduate science education. Nature, 523(7560), p. 282-284, 2015.

BROWN, Anna F. A phenomenological study of undergraduate instructors using the inverted or flipped classroom model. Dissertation (Doctor of Education) - Pepperdine University. 2012. Disponível em: http://pepperdine.contentdm.oclc.org/cdm/ref/collection/p15093coll2/ $\mathrm{id} / 348$. Acesso em: 11/09/2017.

BUSATO, Patrizia; BERRUTO, Remigio; ZAZUETA, Fedro S.; SILVA-LUGO, Jose Lorenzo. Student performance in conventional and flipped classroom learning environments. Applied Engineering in Agriculture, 32(5), p. 509-518, 2016.

CARON CAMBRAIA, Adão; PAGANI BENVENUTTI, Leonardo. Metodologias ativas e tecnologias digitais na formação do professor de computação. Revista Brasileira De Ensino De Ciências E Matemática, 1(1). 2018.

DELOZIER, Sarah J.; RHODES, Matthew G. Flipped Classrooms: a Review of Key Ideas and Recommendations for Practice. Educational Psychology Review, 29(1), p. 141-151, 2016.

DESLAURIERS, Louis; SCHELEW, Ellen; WIEMAN, Carl. Improved learning in a large-enrollment physics class. Science, 332, p. 862-864, 2011.

DRISCOLL, Thomas F. Flipped Learning and democratic Education: The Complete Report. 2012. Disponível em < https://docs.google.com/file/d/0B0VIwE5hKSWta0RqbmdZSGh0WTQ/edit $>$. Acesso em 14/08/2020.

FRANCISCO, Cícero Nestor Pinheiro; OLIVEIRA, Robson Santos. Práticas pedagógicas digitais: o Facebook e a sala de aula invertida na formação dos alunos do ProfLetras. Revista Odisseia, v. 1, n. 2 , p. p. $48-61,6$ set. 2016.

FREEMAN, Scott; EDDY, Sarah L.; MCDONOUGH, Miles; SMITH, Michelle K.; OKOROAFOR, Nnadozie; JORDT, Hannah; WENDEROTH, Mary Pat. Active learning increases student performance in science, engineering and mathematics. Proceedings of The National Academy of Sciences USA. 111(23), p. 8410-8415, 2014.

FREITAS, André Luis Policani; RODRIGUES, Sidilene Gonçalves. Avaliação da confiabilidade de questionário: uma análise utilizando o coeficiente alfa de Cronbach In: Simpósio de Engenharia de Produção, 2005. Disponível em: http://www.simpep.feb.unesp.br/anais/anais_12/ copiar.php?arquivo=Freitas_ALP_A\%20avalia\%E7\%E3o\%20da\%20confiabilidade.pdf. Acesso em 15/04/2017.

GALINDO, Juan Jose; QUINTANA, Maria Graciela Badilla. Innovación docente a través de la metodología flipped classroom: percepción de docentes y estudiantes de educación secundaria. Didasc@lia: Didáctica y Educación, VII, p. 153-172, 2016.

GIANNAKOS, Michail. N.; KROGSTIE, John.; CHRISOCHOIDES, Nikos. Reviewing the flipped classroom research. Proceedings of the Computer Science Education Research Conference on - CSERC', 14, p. 23-29, 2014.

GOMES, Maria Paula Cerqueira; RIBEIRO, Victoria Maria Brant.; MONTEIRO, Dilva Martins.; LEHER, Elizabeth Menezes Teixeira; LOUZADA, Rita de Cássia Ramos. O uso de metodologias ativas no ensino de graduação nas ciências sociais e da saúde: avaliação dos estudantes. Ciência \& Educação (Bauru), Bauru, 16(1), p. 181-198, 2010. 
GÓMEZ, Antonio Garcia. Aprendizaje inverso y motivación en el aula universitaria. Pulso, 39, p. 199-218, 2016.

HAMDAN, Noora; McKNIGHT, Patrick; McKNIGHT, Katherine.; ARFSTROM, Kari. M. white paper based on the literature review titled a review of flipped learning. Flipped Learning Network, Pearson, George Mason University, 2013. Disponível em: https:/flippedlearning.org/ wp-content/uploads/2016/07/WhitePaper_FlippedLearning.pdf.

HUBER, Elaine; WERNER, Ashleigh. A review of the literature on flipping the STEM classroom: preliminary findings. Show Me The Learning. Proceedings Ascilite. Adelaide: Ascilite, 2016.

JENSEN, Jamie L.; KUMMER, Tyler A.; GODOY, Patricia D. D. M. Improvements from a flipped classroom may simply be the fruits of active learning. CBE - Life Sciences Education, Spring, 14, p. 1-12, 2015.

JOHNSON, Grahan Brent. Student perceptions of the flipped classroom. 2013. MA thesis - University of British Columbia, Canadá, 2013.

JOHNSON, Larry; ADAMS BECKER, Samantha; ESTRADA, V.; FREEMAN, A. NMC Horizon Report: 2014. Higher Education Edition. Austin, Texas: The New Media Consortium, 2014.

KARABULUT-ILGU, Aliye; JARAMILLO CHERREZ, Nadia; JAHREN, Charles T. A systematic review of research on the flipped learning method in engineering education. British Journal of Educational Technology, 49(3), p. 298-411, 2017.

KIM, Kyung Yeul; KIM, Yong. What are learning satisfaction factors in flipped learning? Advances in Computer Science and Ubiquitous Computing. CSA 2016, CUTE 2016, UCAWSN 2016. Lecture Notes in Electrical Engineering, vol. 421, 2017.

LAGE, Maureen J.; PLATT, Gleen J.; TREGLIA, Michael. Inverting the classroom: a gateway to creating an inclusive learning environment. Journal of Economic Education, 3(1), p. 30-43, 2000 .

LEITE, Sérgio Antônio da Silva. Dimensões afetivas na relação professor-aluno. In: Tassoni, Elvira C. M. A afetividade em sala de aula: as condições de ensino e a mediação do professor. São Paulo: Ed. Casa do psicólogo, p. 18-34, 2006.

LYMAN, Frank. Think-Pair Share: An expanding teaching technique: MMA-CIE. Cooperative News, 1, p. 1-2, 1987.

MARLOWE, Cara A. The Effect of the Flipped Classroom On Student Achievement and stress. Master's thesis - Montana State University, 2012. Disponível em http://scholarworks.montana.edu/xmlui/bitstream/handle/1/1790/MarloweC0812.pdf?sequence=1. Acesso em 15/03/2015.

MAZUR, Eric. Peer Instruction: a user's manual. Upper Saddle River: Prentice Hall, 1997.

MAZUR, Eric. Farewell, Lecture? Science, 323, p. 50-51, 2009.

MAYER, Veronica Feder. Aplicações do Método caso em Sala de Aula. Rio de Janeiro: Fundação Getúlio Vargas, 2012.

MCNALLY, Brenton; CHIPPERFIELD, Janine; DORSETT, Pat; DEL FABBRO, Letitia; FROMMOLT, Valda; GOETZ, Sandra; LEWOHL, Joanne; MOLINEUX, Matthew; PEARSON, Andrew; REDDAN, Gregory; ROIKO, Anne; RUNG, Andrea. Flipped classroom experiences: student preferences and flip strategy in a higher education context. Higher Education, 73(2), p. 281-298, 2017. 
MENNELLA, Thomas A. Comparing the efficacy of flipped vs. alternative active learning in a college genetics course. The American Biology Teacher, 78(6), p. 471-479, 2016.

MICHAEL, Joel. Where's the evidence that active learning works? Advances Physiology Education, 30, p. 159-167, 2006.

MOREIRA, Marco Antonio. Metodologias de pesquisa em ensino. São Paulo: Livraria da Física. 1ํㅡㄹ edição. 2011.

MUSALLAM, Ramsey. The effects of using screencasting as a multimedia pre-training tool to manage the intrinsic cognitive load of chemical equilibrium instruction for advanced high school chemistry students. São Francisco, Califórnia, Estados Unidos: The University of San Francisco. Dissertation (Doctor of Education). 2010. Disponível em < https://search.proquest.com/ docview/742560032 > Último acesso: 11/08/2020.

NOURI, Jalal. The flipped classroom: for active, effective and increased learning - especially for low achievers. International Journal of Educational Technology in Higher Education, 13(1), p. 1-10, 2016.

NOVAK, Gregor M.; PATTERSON, Evelyn T.; GAVRIN, Andrew D.; CHRISTIAN, Wolfgang. Just-in-time teaching: blending active learning with web technology. Upper Saddle River: Prentice Hall, 1999.

O'FLAHERTY, Jacqueline; PHILLIPS, Craig. The use of flipped classrooms in higher education: a scoping review. Internet and Higher Education, 25, p. 85-95, 2015.

OGDEN, Lori. Student Perceptions of the Flipped Classroom in College Algebra. Primus, 25:910, p. 782-791, 2015.

OLAKANMI, Eunice. Eyitayo. The Effects of a Flipped Classroom Model of Instruction on Students' Performance and Attitudes Towards Chemistry. Journal of Science Education and Technology, 26(1), p. 127-137, 2017.

OFUGI, Mariana Santana. A sala de aula invertida como técnica alternativa de ensino: um enfoque no desenvolvimento da autonomia do aprendiz de inglês como L2/LE. Dissertação (Mestrado) - Universidade Federal de Goiás, Faculdade de Letras (FL), Programa de Pós-Graduação em Letras e Linguística, Goiânia, 2016.

PAPADOPOULOS, Cristopher; ROMAN, Aidsa Santiago. Implementing an inverted classroom model in engineering statics: Initial results. Proceedings of the 40th ASEE/IEEE Frontiers in Education Conference. Washington DC: American Society for Engineering Statistics, 2010.

PAVANELO, Elisangela; LIMA, Renan. Sala de aula invertida: a análise de uma experiência na disciplina de Cálculo I. Bolema, Rio Claro, SP, 31(58), p. 739-759, 2017.

ROCHA, Henrique Martins; LEMOS, Washington de Macedo. Metodologias ativas: do que estamos falando? Base conceitual e relato de pesquisa em andamento. Simpósio Pedagógico e Pesquisas em Comunicação, Resende - RJ, 9, 2014.

SENGEL, Erhan. To Flip or not to flip: Comparative case study in higher education in Turkey. Computers in Human Behavior, 64, p. 547-555, 2016.

SERQUEIRA, Caroline Ferreira Costa. A sala de aula invertida no contexto da educação básica possibilidades de mudança na prática docente. Curitiba, Paraná: Pontifícia Universidade Católica do Paraná. Dissertação de Mestrado: Programa de Pós-Graduação em Educação. 2017. 
SLETTEN, Sarah Rae. Investigating Flipped Learning: Student Self-Regulated Learning, Perceptions and Achievement in an Introductory Biology Course. Journal of Science Education and Technology, 26, p. 347, 2017.

STRAYER, Jeremy. How learning in an inverted classroom influences cooperation, innovation and task Orientation. Learning Environments, 15(2), p. 171, 2012.

SUHR, Inge Renate Frose. Desafios no uso da sala de aula invertida no Ensino Superior. Transmutare, 1(1), p. 4-21, 2016.

TAZIJAN, Farina Nozakiah; ABDULLAH, Che Haslina; ZAINOL, Noorliza; NOOR, Syuhirdy Mat; JOHARI, Noorsa Riza. Building communication skills through flipped classroom. International Academic Research Journal of Social Science, 3(1), p. 142-147, 2017.

TREVELIN, Ana Tereza Colenci; PEREIRA, Marco Antonio Alves; OLIVEIRA NETO, José Dutra. A utilização da "sala de aula invertida" em cursos superiores de tecnologia: comparação entre o modelo tradicional e o modelo invertido "flipped classroom" adaptado aos estilos de aprendizagem. Revista de Estilos de Aprendizagem, Madrid, 11(12), p. 137-150, 2013.

TURAN, Zeynep; GOKTAS, Yuksel. The flipped classroom: Instructional efficiency and impact on achievement and cognitive load levels. Journal of E-Learning and Knowledge Society, 12(4), p. 51-62, 2016.

VALENTE, José Armando. Blended learning e as mudanças no Ensino Superior: a proposta da sala invertida. Educar em Revista, Curitiba, Brasil, Edição Especial, 4, p. 79-97, 2014.

VALÉRIO, Marcelo. Autonomia de professores na sala de aula invertida: uma análise sobre a profissionalidade e a racionalização da prática docente. Maringá, Paraná: Universidade Estadual de Maringá. Tese (Doutorado em Educação para a Ciência e a Matemática). 2018. Disponível em < https://sucupira.capes.gov.br/sucupira/public/consultas/coleta/trabalhoConclusao/viewTrabalhoConclusao.jsf?popup=true\&id_trabalho=7198632\# > Acesso em 13/03/2019.

VALÉRIO, Marcelo; BELETI Junior, Carlos R. Caracterização da produção acadêmica brasileira sobre a sala de aula invertida. ACTIO, Curitiba, v. 4, n. 3, p. 17-34, set./dez. 2019. Disponível em: <https://periodicos.utfpr.edu.br/actio>. Acesso em: 10/08/2019.

VALÉRIO, Marcelo.; MOREIRA, Lúcia Olivo Rosas Moreira. Sete críticas à sala de aula invertida. Revista Contexto \& Educação, 33(106), p. 215-230, 2018.

VALÉRIO, Marcelo; MOREIRA, Ana Lucia Olivo Rosas.; BRAZ, Barbára Cândido.; NASCIMENTO, William Junior do. A sala de aula invertida na universidade pública Brasileira: evidências da prática em uma licenciatura em ciências exatas. Revista Thema. vol. 16, p. 195-211, 2019.

VIEIRA, Sonia. Como elaborar questionários. São Paulo: Atlas, 2009.

WALDROP, Mitchell M. The science of teaching sciences. Nature, 523(7560), p. 272-274, 2015.

ZAINUDDIN, Zamzami; HALILI, Siti Hajar. Flipped Classroom Research and Trends from Different Fields of Study. International Review of Research in Open and Distributed Learning, 17(3), p. 313-340, 2016. 


\section{ANEXO I}

\section{Questionário de Avaliação Sala de Aula Invertida}

QUESTIONÁRIO DE AVALIAÇÃO DA SALA DE AULA INVERTIDA - UFPR Jandaia - 2017.1

1) Seleclone a(s) disclplina(s) que vocé cursou em 2017-1, na(s) qual(Is) vivenclou o modelo "sala de aula invertida":

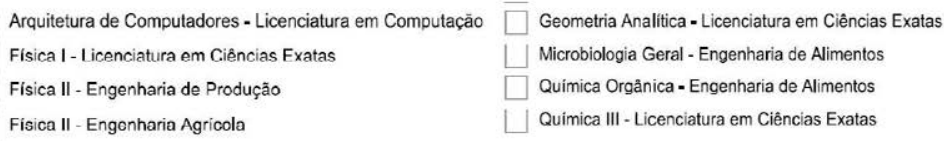

2) Foi a primeira vez que vocè cursou uma disciplina com essa proposta (sala de aula invertida)?

$\square \mathrm{SIM} \bigcirc \mathrm{NĀO}$

3) Como você avalia a ideia de que o aluno estude antecipadamente os conteúdos, e que o o tempo de aula seja usado para a resolução de exercícios e problemas com os seus colegas e sob orientação do professor?

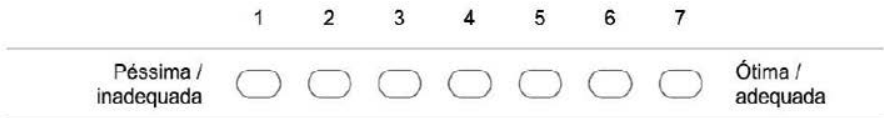

4) Você percebeu haver mais e melhor interação entre os estudantes; e, desses, com o professor?

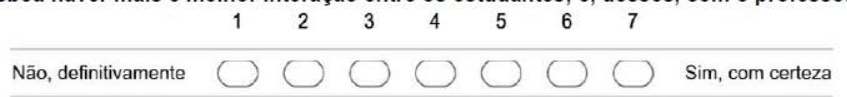

5) Você se sentlu mals motlvado/a a frequentar e particlpar das aulas?

$\begin{array}{lllllllll} & 1 & 2 & 3 & 4 & 5 & 6 & 7 & \\ \text { Não, definitivamente } & \bigcirc & \bigcirc & \circlearrowleft & \bigcirc & \bigcirc & \bigcirc & \bigcirc & \text { Sim, com certeza }\end{array}$

6) Seu tempo total de estudo (tanto dentro como fora da aula) foi maior que nas aulas tradicionais?

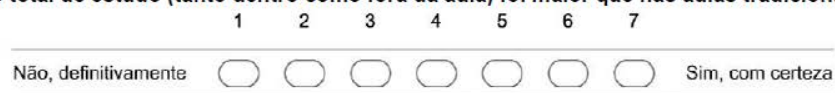

7) Com que frequência você estudou previamente, conforme orientado pelo professor, os vídeos, notas de aula e outros materiais?

\begin{tabular}{llllllll} 
& 1 & 2 & 3 & 4 & 5 & 6 & 7 \\
Nunca $\square$ & $\square$ & $\square$ & $\square$ & & \\
\hline
\end{tabular}

8) O estudo prévio (antes da aula em sala) permitiu que você compreendesse e dominasse melhor os conteúdos?

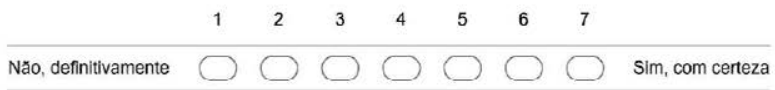

9) As metodologias utilizadas em sala (ex. resolução guiada de problemas, atividades de discussão em grupos, quizzes, desafios práticos) permitiram que você compreendesse e dominasse melhor o conteúdo?

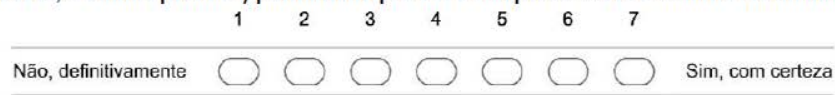




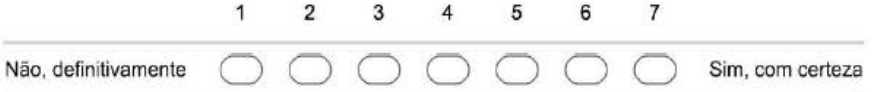

11) Os vídeos, notas de aula e outros materiais encaminhados pelos professores estavam adequados às suas capacidades de aprendizagem?

$\begin{array}{lllllllll} & 1 & 2 & 3 & 4 & 5 & 6 & 7 & \\ \text { Năo, definitivamente } \bigcirc & \bigcirc & \bigcirc & \bigcirc & \bigcirc & \bigcirc & \bigcirc & \text { Sim, com certeza }\end{array}$

12) Você avalia que seu desempenho na disciplina foi compativel com a sua capacidade, compromisso e dedicação durante o semestre?

$\begin{array}{lllllllll} & 1 & 2 & 3 & 4 & 5 & 6 & 7 & \\ \text { Não, definitivamente } \bigcirc & \bigcirc & \bigcirc & \bigcirc & \bigcirc & \bigcirc & \bigcirc & \bigcirc & \bigcirc\end{array}$

13) As formas e os critérios de avaliação utilizados na disciplina foram justos e adequados?

$\begin{array}{lllllllll} & 1 & 2 & 3 & 4 & 5 & 6 & 7 & \\ \text { Năo, definitivamente } \square & \bigcirc & \bigcirc & \bigcirc & \bigcirc & \bigcirc & \bigcirc & \text { Sim, com certeza }\end{array}$

14) Em termo gerais, como você avalia a disciplina que cursou? (Atribua uma nota entre 0 e 10)

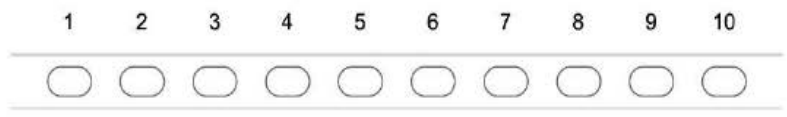

15) 0 que você mais gostou como aluno de uma "sala de aula invertida"? (pense nos benefícios você percebeu?)

16) Que sugestóes você daria para melhorar a sala de aula invertida? (pense nas desvantagens que você percebeu).

17) Depois dessa experiência como aluno/a em uma SALA DE AULA INVERTIDA, gostaria de ter outras aulas e disciplinas no mesmo modelo?

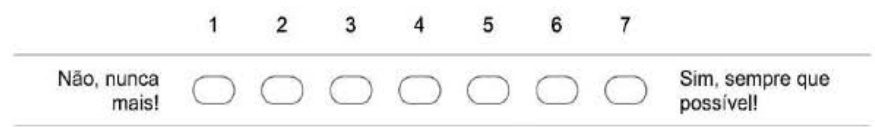

